The Astrophysical Journal, 461:72-83, 1996 April 10

(C) 1996. The American Astronomical Society. All rights reserved. Printed in U.S.A.

\title{
HUBBLE SPACE TELESCOPE OBSERVATIONS OF THE LUMINOUS IRAS SOURCE FSC 10214+4724: A GRAVITATIONALLY LENSED INFRARED QUASAR ${ }^{1}$
}

\author{
Peter R. Eisenhardt, ${ }^{2}$ Lee Armus, ${ }^{3}$ David W. Hogg, ${ }^{3}$ B. T. Soifer, ${ }^{3}$ \\ G. NeUgebauer, ${ }^{3}$ AND Michael W. WeRner ${ }^{2}$ \\ Received 1995 July 17; accepted 1995 October 12
}

\begin{abstract}
With a redshift of 2.3 , the $I R A S$ source FSC $10214+4724$ is apparently one of the most luminous objects known in the universe. We present an image of FSC $10214+4724$ at $0.8 \mu$ m obtained with the Hubble Space Telescope (HST) WFPC2 Planetary Camera. The source appears as an unresolved $(<0$ ".06) arc 0"7 long, with significant substructure along its length. The center of curvature of the arc is located near an elliptical galaxy $1^{\prime \prime} .18$ to the north. An unresolved component 100 times fainter than the arc is clearly detected on the opposite side of this galaxy. The most straightforward interpretation is that FSC $10214+4724$ is gravitationally lensed by the foreground elliptical galaxy, with the faint component a counterimage of the IRAS source. The brightness of the arc in the $H S T$ image is then magnified by $\sim 100$, and the intrinsic source diameter is $\sim 0$.01 $(80 \mathrm{pc})$ at $0.25 \mu \mathrm{m}$ rest wavelength. The bolometric luminosity is probably amplified by a smaller factor $(\sim 30)$ as a result of the larger extent expected for the source in the far-infrared. A detailed lensing model is presented that reproduces the observed morphology and relative flux of the arc and counterimage and correctly predicts the position angle of the lensing galaxy. The model also predicts reasonable values for the velocity dispersion, mass, and mass-tolight ratio of the lensing galaxy for a wide range of galaxy redshifts. A redshift for the lensing galaxy of $\sim 0.9$ is consistent with the measured surface brightness profile from the image, as well as with the galaxy's spectral energy distribution. The background lensed source has an intrinsic luminosity $\sim 2 \times 10^{13} L_{\odot}$ and remains a highly luminous quasar with an extremely large ratio of infrared to optical/ultraviolet luminosity.
\end{abstract}

Subject headings: gravitational lensing — infrared: galaxies - galaxies: individual (FSC 10214+4724)

\section{INTRODUCTION}

Ever since its identification with a redshift 2.286 optical emission-line source by Rowan-Robinson et al. (1991), leading to an inferred bolometric luminosity $\sim 5 \times 10^{14}$ $L_{\odot}$, the IRAS source FSC $10214+4724$ has been the subject of enormous attention. Detections of CO (Brown \& Vanden Bout 1991; Solomon, Downes, \& Radford 1992; Tsuboi \& Nakai 1992) and submillimeter continuum emission (Clements et al. 1992; Downes et al. 1992) from the source confirmed the presence of huge quantities of gas and dust. With a vastly larger lookback time and luminosity than any other known IRAS source, FSC $10214+4724$ appeared to be either an extremely luminous dustembedded quasar or a representative of a new class of astronomical object, e.g., a primeval galaxy.

However, while the redshift of the IRAS source is secure, its intrinsic luminosity is less certain. The fact that FSC $10214+4724$ lies at the flux limit of the IRAS survey, combined with the presence of several red companion objects within a few arcseconds, led Elston et al. (1994) to suggest that the IRAS source might be gravitationally lensed by a foreground group of galaxies. Intriguingly, Matthews et al. (1994) found arcs emerging from the source in a deconvolved $K$-band image with 0 ".6 seeing taken with the Keck

\footnotetext{
${ }^{1}$ Based on observations made with the NASA/ESA Hubble Space Telescope, obtained at the Space Telescope Science Institute, which is operated by AURA, Inc., under NASA contract NAS 5-26555.

2 MS 169-327, Jet Propulsion Laboratory, California Institute of Technology, 4800 Oak Grove Drive, Pasadena, CA 91109.

${ }^{3}$ Division of Physics, Math, and Astronomy, California Institute of Technology, Pasadena, CA 91125.
}

telescope. Matthews et al. considered the lensing hypothesis but concluded it was unlikely because the image morphology was not achromatic. Broadhurst \& Lehár (1995) modeled the source as gravitationally lensed, finding support for their model from a reanalysis of the Matthews et al. data. Graham \& Liu (1995) also argue for lensing, based on deconvolution of a more recent $(1995 \mathrm{March})$ Keck $K$-band image with 0.4 seeing. Trentham (1995) argues on statistical grounds that magnification due to lensing is likely to be less than a factor of 10 , although larger magnifications are reasonable for smaller far-IR source sizes than the $1 \mathrm{kpc}$ Trentham assumed.

We present an image of FSC 10214+4724 taken in 1994 December at $8000 \AA$ with the HST WFPC2 Planetary Camera with 0 ". 1 resolution. This image provides dramatic support for the lensing hypothesis, implying a magnification in the $H S T$ data of $\sim 100$. We use the image to derive a detailed model for the intrinsic properties of the lensed source and the lensing galaxy.

For reference, at the FSC $10214+4724$ redshift $z=2.286$, one 0"0455 Planetary Camera pixel subtends $300(180) h^{-1}$ pc for $q_{o}=0(0.5)$, while these values are $239(191) h^{-1} \mathrm{pc}$ for $z=0.9$, where $h \equiv H_{0} / 100 \mathrm{~km} \mathrm{~s}^{-1} \mathrm{Mpc}^{-1}$. Where not otherwise specified, we assume $H_{0}=50 \mathrm{~km} \mathrm{~s}^{-1} \mathrm{Mpc}^{-1}$ and $q_{0}=0.5$.

\section{OBSERVATIONS AND REDUCTION}

Three frames, each $2200 \mathrm{~s}$ long, were obtained on consecutive orbits with the WFPC2 F814W filter on 1994 December 10 and 11 (UT). FSC $10214+4724$ was positioned near the center of the Planetary Camera, and each exposure was displaced from the other two by an integer 
number ( 5 or 10$)$ of $\mathrm{PC}$ pixels in both axes. The Wide Field Camera data are not considered here.

After standard processing provided by STScI, the multiple frames were used to filter out cosmic rays and hot pixels. Although these defects are quite prominent and affect $\sim 4 \%$ of the pixels in each frame, the main characteristics of the combined image discussed in $\S 3.1$ are discernible in each frame even without this filtering.

Cross-correlations were performed on pairs of frames to confirm that the actual displacements between frames, as measured in pixels, were integers to within 0.2 pixels. The frames were then trimmed by the appropriate number of rows and columns to coregister them, and the STSDAS task CRREJ was used to average them together, iteratively excluding pixels that deviated from the previous iteration's average value by more than $3 \sigma$. The minimum value at each pixel location was used for the initial estimate of the average, and $\sigma$ was the value expected from Poisson statistics and the gain and read noise. To remove multiple pixel cosmic-ray events, a stricter limit of $1.5 \sigma$ was applied to the 4 pixels adjacent to any pixel that exceeded the $3 \sigma$ criterion. Finally a median filtering routine was applied to identify and interpolate over a few dozen isolated pixels that deviated sharply from their neighbors in the average image, presumably because they were corrupted in all three frames. None of these latter pixels fall within objects in the field, and only a handful of the pixels in the components discussed below are based on data from less than two frames.

\section{RESULTS}

The combined image of the full Planetary Camera field is shown in Figure $1 a$ (Plate 1), while Figures $1 b$ and $1 c$ show the FSC $10214+4724$ region in progressively greater detail.

A synthetic point spread function (PSF) derived from the "Tiny Tim" HST image modeling software package was used to deconvolve the average image because a good empirical point spread function was not available (see $\S 3.2$.). The synthetic PSF was calculated for a source with the color of a K-star in F814W at the location of FSC $10214+4724$ in the Planetary Camera field. Figure $1 d$ shows the same region covered in Figure $1 c$ after a mild deconvolution of the data (10 iterations of the STSDAS implementation of the Lucy-Richardson algorithm) onto a grid subsampled 4 times more finely than the original pixels.

\subsection{Morphology}

At the resolution of the Planetary Camera, an arclike structure dominates the morphology of the emission-line source. In the terminology of Matthews et al. (1994), which is adopted here, the arclike structure is component 1 (see Fig. $1 b$ ). The extent of this arc is smaller than shown in Matthews et al., and there is a sharply defined ridge of high surface brightness emission which is 0 . 7 long and essentially unresolved in the transverse direction. Lower surface brightness emission can be seen extending the arc $\sim 0$. 4 to the west, and a similar amount (but at a considerably fainter level) to the east-northeast. There is also a hint of still fainter emission extending a few tenths of an arcsecond due east (not along a circular arc) from the eastern tip of the bright ridge. Within the bright ridge are at least two peaks separated by 0 .'24, with the brighter peak toward the east. The center of curvature of the arc was fitted and found to be $\sim 0$ ". 12 west-northwest of the center of component 2 (which is $1^{\prime \prime} .18$ from the arc). Component 2 has a smooth light distribution that is resolved and slightly elongated (see $\S \S 3.2$ and 4.3). Directly opposite component 2 from the arc is a faint but clearly visible source (component 5 in Fig. 1b), 0 .43 from the center of component 2 . Component 3 is resolved and has a feature that is suggestive of a tidal arm leading back toward component 2. Component 4 appears to be a highly inclined galaxy.

\subsection{Brightness Profiles}

In an attempt to quantify the radial extent of the arc, pixels from the sector subtended by the brightest 0.5 of the arc at component 2 were sorted in order of radius from component 2 . To reduce the effect of the tangential substructure along the arc, a running average of the flux from 5 pixels in this radially sorted list was calculated. Figure 2 plots this running average flux as a function of the average radius of those pixels less the 1 "18 distance of component 1 from component 2 . For comparison, the (unaveraged) radial profiles are plotted for stars $\mathrm{A}$ and $\mathrm{H}$ (see Fig. 1a), for components 2 and 5, and for the synthetic PSF that was used in the deconvolution shown in Figure $1 d$.

While the wings of the synthetic PSF fall inside those of the arc, the empirical PSFs of stars A (outside its saturated core) and $\mathrm{H}$ match the arc cross section reasonably well. It therefore appears likely that the synthetic PSF underestimates the FWHM of the true PSF. Based on the synthetic PSF, we estimate an upper limit of 0 ."06 (500 pc) for the intrinsic FWHM of the arc in the radial direction. Note that the effects of the running average, of any error in using component 2 as the center of the arc, and of the smaller size of the synthetic PSF all work in the direction of leading us to overestimate this dimension.

The deconvolved image shown in Figure $1 d$ also yields a 0"06 FWHM for the arc, but this holds true for star $\mathrm{H}$ after deconvolution as well. Because the individual frames are separated by integer numbers of PC pixels, there is little leverage on finer scale structure. Deconvolution does emphasize the high surface brightness of the arc, however, increasing it by a factor of 3 .

In short, we see no evidence that component 1 is resolved in the radial direction. (In $\S 4.1$ we will argue that the intrinsic FWHM of the arc is $\sim 0$. 01 ). Component 5 also appears unresolved, although its profile suffers from much lower signal to noise.

Component 2, however, is clearly resolved in Figure 2. To extend the measurement of component 2's surface brightness profile to larger radii, the image was rotated $180^{\circ}$ about the center of component 2 , and pixels at the locations of other objects in the original image were replaced with pixels from the rotated image. This assumes elliptical symmetry for component 2 in the replaced regions, which cover a maximum of $25 \%$ (at $r=1.3$ ) of the area at any radius, and $7 \%$ of the total area. Figure 3 shows the resulting radial surface brightness profile for component 2 . A de Vaucouleurs profile with an effective radius $r_{e} \approx 1$ ".3 $(10 \mathrm{kpc})$ provides a much better fit to component 2 than do exponential disk models, which suggests that this object is an early-type galaxy. The measured ellipticity of component 2 inside the arc is $\approx 0.16 \pm 0.1$ at a position angle of $\approx 3^{\circ} \pm 15^{\circ}$ east of north. Excess surface brightness appears near a radius of 1 ."4 even though the component 1 pixels (which are near this radius) have been replaced. As a check, the surface brightness profile was measured within sectors centered on component 2 from position angles $73^{\circ}-133^{\circ}$ and $233^{\circ}-318^{\circ}$, 


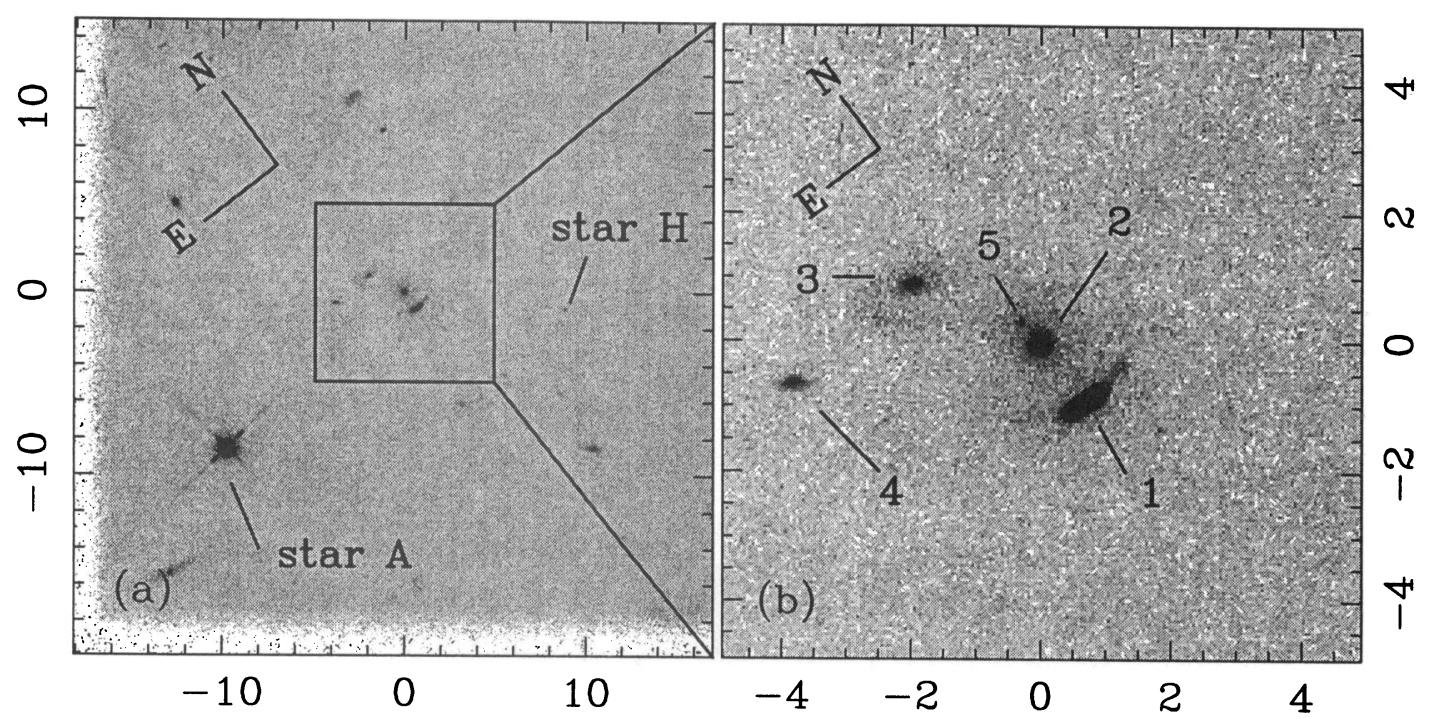

PLATE 1

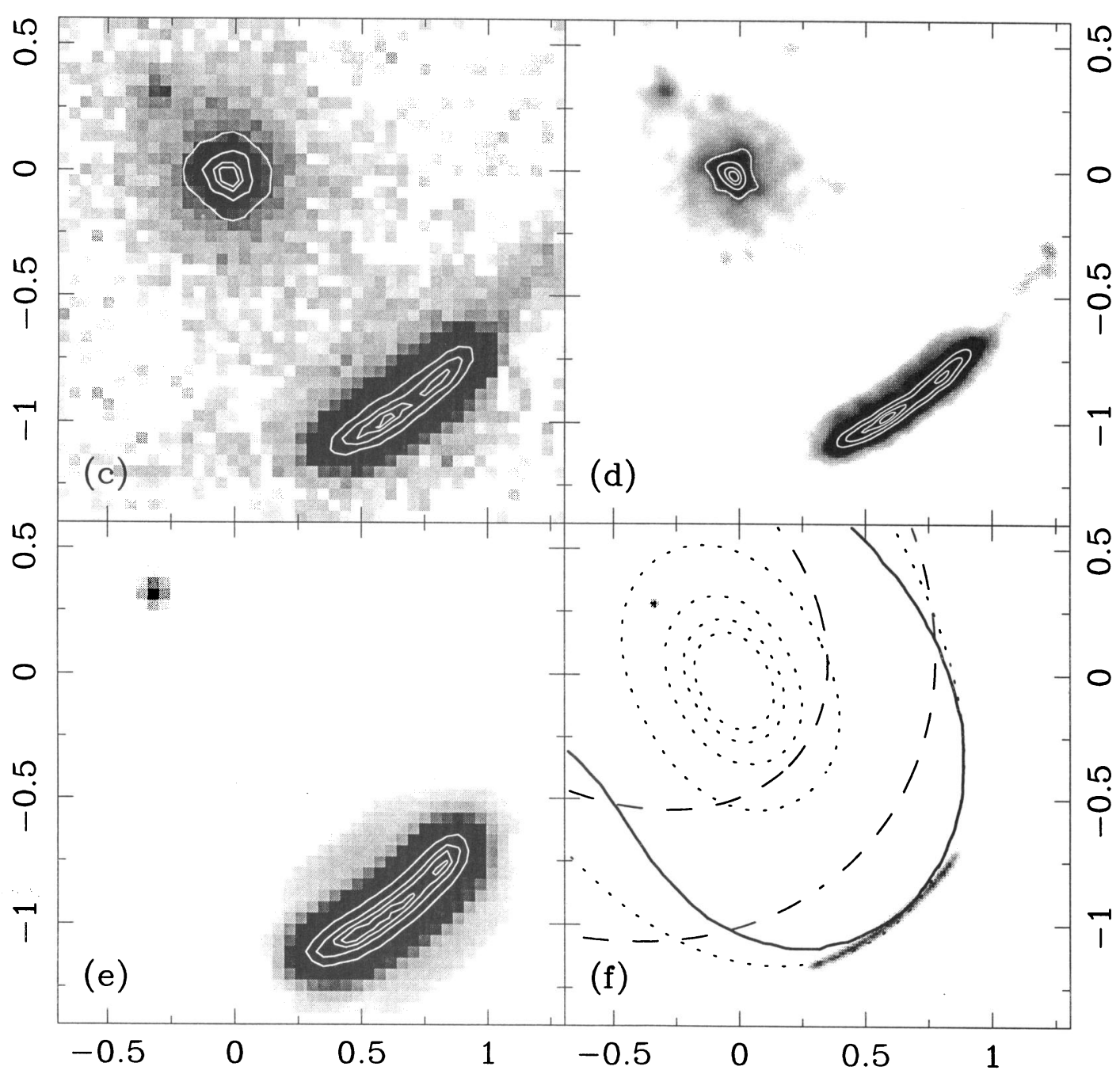

FIG. 1.- Montage of HST Planetary Camera (PC) imaging of IRAS FSC $10214+4724$ in F814W. Panels $a, b$, and $c$ show the image at progressively finer scales, as indicated by the axes, which are labeled in arcseconds relative to component 2 (see panel $b$ ). Panels $d, e$, and $f$ have the same scale and center as panel $c$. Panel $d$ is a partially deconvolved version of the data with a factor of 4 subsampling. Panel $e$ shows the predicted image configuration given the lens model and a uniform circular disk source of radius 0.0055 . The image configuration has been convolved with the synthetic HST PSF and binned into PC pixels to allow direct comparison with panel $c$. Panel $f$ shows further details of the lens model: as in panel $d$ the pixel size is 4 times smaller, and the model image has been lightly smoothed to a FWHM of 0 ".02. Lines indicate contours of mass density (dotted line), potential (dashed line), and the critical curve (solid line) for the model. The gray levels in $(f)$ range linearly from zero (white) to the peak value in the arc (black), but have been enhanced (in $f$ only) by a factor of 7 at the counterimage location. The gray levels in $(a-e)$ range linearly from $0.5 \%$ to $5 \%$ of the peak brightness in component $1\left(\mu_{814}=17.6 \mathrm{mag}\right.$ arcsec ${ }^{-2}$ in panels $a-c, \mu_{814}=13.6 \mathrm{mag} \operatorname{arcsec}^{-2}$ in panel $\left.d\right)$. Contour levels in $(c)-(e)$ are at $25 \%, 50 \%, 75 \%$, and $90 \%$ of this peak brightness for component 1, or at $25 \%, 50 \%$, $75 \%$, and $90 \%$ of the peak brightness for component $2\left(\mu_{814}=19.4 \mathrm{mag} \mathrm{arcsec}^{-2}\right.$ in panel $c, \mu_{814}=15.6 \mathrm{mag}$ arcsec ${ }^{-2}$ in panel $\left.d\right)$, as appropriate. North is 37.1 counterclockwise from vertical in all panels, with east $90^{\circ}$ counterclockwise from north, as shown in panels $a$ and $b$.

EISENHARDT et al. (see 461,73 ) 


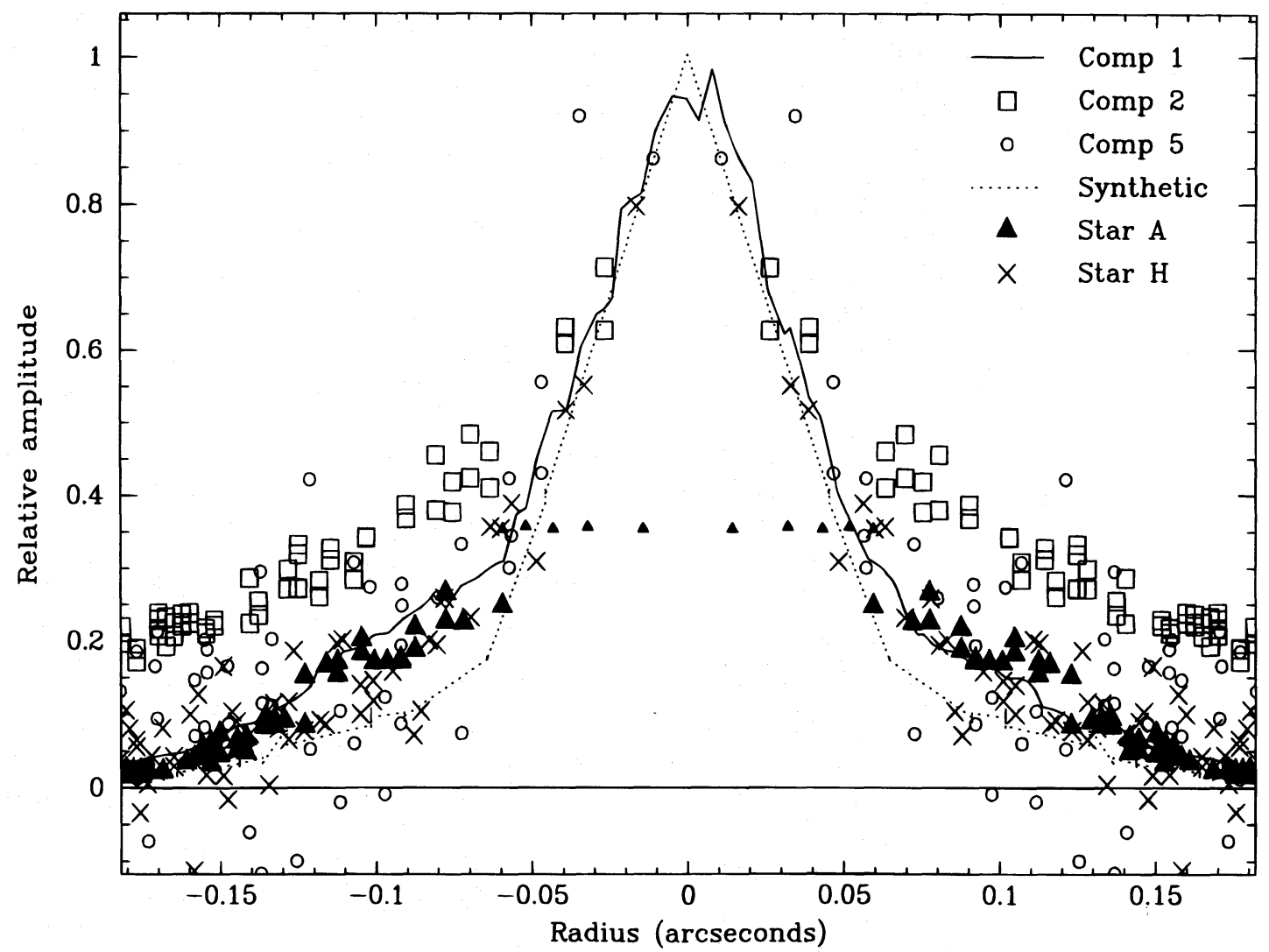

Fig. 2.-Radial profiles for objects identified in Figs. $1 a$ and $1 b$. Component 1 appears unresolved relative to stars A and $\mathrm{H}$. For component 1 the equivalent radial profile is plotted, as discussed in § 3.2. The synthetic PSF was used to generate the deconvolution shown in Fig. 1d. The vertical scales for the profiles were normalized at the smallest radius available, except for star A, whose core is saturated in our image, and which was normalized to the synthetic PSF at the first radius which was not saturated. The data points plotted for negative radii are identical to those for positive radii except for component 1 .

angles that bypass all obvious emission sources in Figure $1 b$. The value for $r_{e}$ in this case was 1 ".0 (a smaller $r_{e}$ is consistent with these sectors being along the minor axis), and excess light was again found near $1^{\prime \prime} 4$ radius. The total excess light at this radius is very roughly equivalent to a magnitude 23 source.

\subsection{Photometry}

Photometric measurements obtained from the Planetary Camera image for the components are given in Table 1. One count in the image corresponds to $1.185 \times 10^{-21} \mathrm{ergs} \mathrm{cm}^{2}$ $\mathrm{s}^{-1} \AA^{-1}$ or to a magnitude of 30.00 in the $\mathrm{F} 814 \mathrm{~W}$ band with Vega set to magnitude 0 . From the measured standard deviation per pixel, the sensitivity limit $(3 \sigma)$ is $m_{814} \sim 28.2$ mag for a point source or $\mu_{814} \sim 25.6 \mathrm{mag} \operatorname{arcsec}^{-2}$. Positions are relative to component 2 , whose position in the HST guide star system is given in Table 1. Polygonal apertures were used to include the faint emission seen extending from components 1 and 3 . The flux for component 5 was measured using a 0 ". 35 diameter aperture, with the local background measured using the mode of an annulus of width 0 ."1 surrounding this aperture, and corrected for PSF losses using the star $\mathrm{H}$ curve of growth. This flux was checked by subtracting away the image rotated $180^{\circ}$ about component 2 and also by subtracting the elliptical model fit to component 2 discussed in $\S 3.2$. All three methods consis-

TABLE 1

Photometry of ObJects IN $H S T$ F814W IMAGe of IRAS FSC $10214+4724$

\begin{tabular}{|c|c|c|c|c|}
\hline Component & $m_{814}$ & $\Delta \alpha$ & $\Delta \delta$ & Comment \\
\hline & 20.44 & 0.10 & -1.16 & Includes faint extensions \\
\hline 2. & $\begin{array}{l}21.41 \\
20.3\end{array}$ & 0 & 0 & $\begin{array}{l}\text { Inside } r=0.85 \\
\text { Total }\end{array}$ \\
\hline ........... & $\begin{array}{l}23.16 \\
22.98\end{array}$ & $1: 03$ & 1.93 & $\begin{array}{l}\text { Inside } r=0.5 \\
\text { Including component to east }\end{array}$ \\
\hline$\ldots$ & 23.58 & 3.42 & 1.79 & $\sim 1^{\prime \prime} 1$ wide $\times 0$ ". 6 high polygonal aperture \\
\hline$\ldots$ & 25.5 & 0.03 & 0.43 & Inside $r=0.35$ \\
\hline Star $\mathrm{H} \ldots \ldots$ & 24.54 & -6.59 & -6.20 & $\ldots$ \\
\hline
\end{tabular}

Note.-Components are identified in Fig. $1 a$ and $1 b$. Positions are with respect to the center of component 2 , which is approximately $\alpha=10^{\mathrm{h}} 24^{\mathrm{m}} 34^{8} .56, \delta=47^{\circ} 09^{\prime} 10^{\prime \prime} 8,(\mathrm{~J} 2000)$ in the HST guide star catalog frame. For component 1 the position is for the peak brightness. 


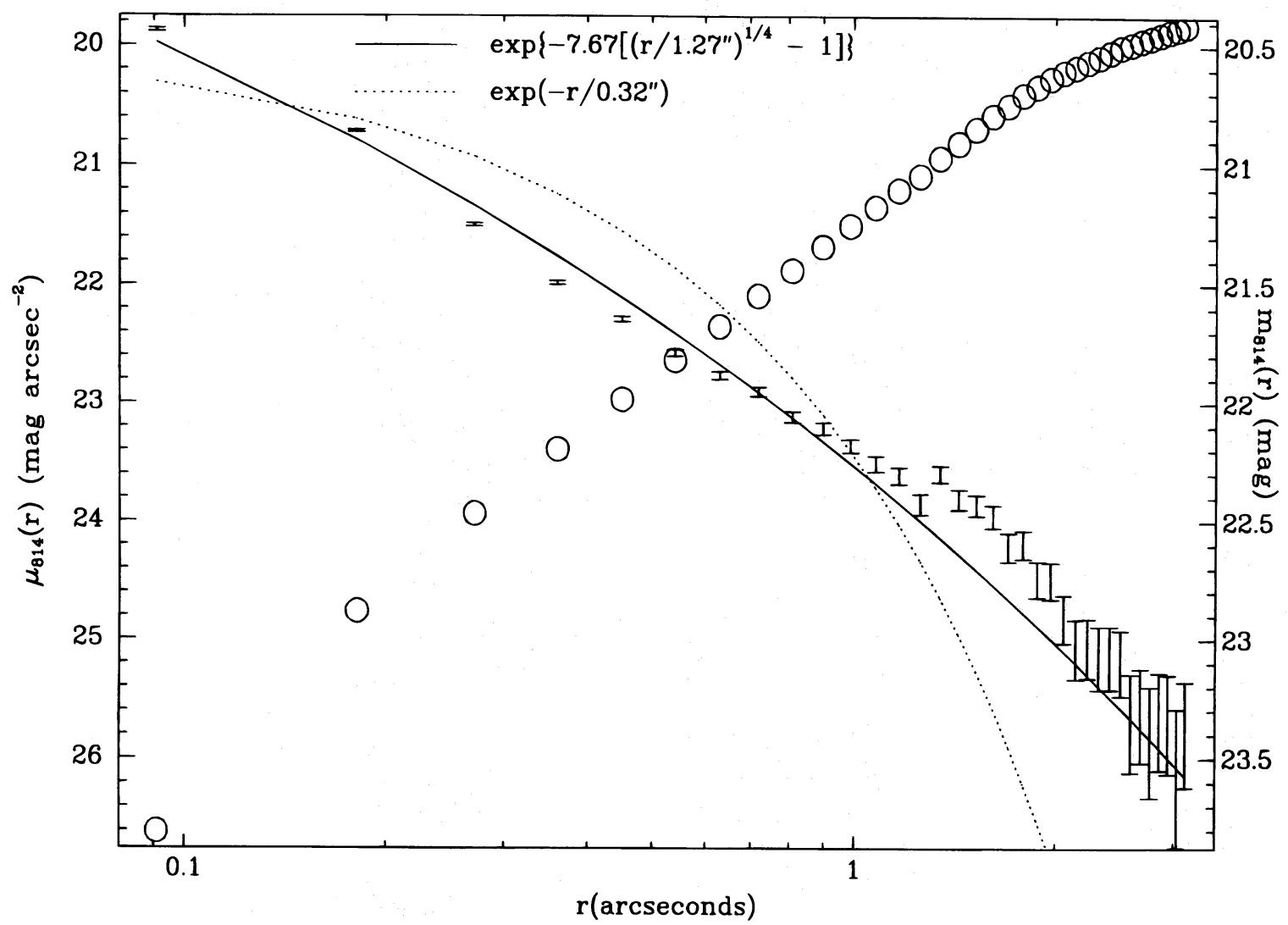

Fig. 3.-The surface brightness of component 2 is plotted as a function of radius, together with a de Vaucouleurs profile with $r_{e}=1$."27 (solid line) and an exponential profile with $r_{d}=0.32$ (dotted line). Magnitude as a function of aperture radius is shown by the open circles and the right-hand scale. The magnitude appears to converge near $m_{814}=20.3 \mathrm{mag}$, yielding a consistent value for $r_{e}$.

tently gave a value close to 100 for the flux ratio of component 1 to component 5 , and we adopt 100 for this important ratio for the remainder of the paper.

\section{DISCUSSION}

The morphology of the components of FSC $10214+4724$, a circular arc (component 1 ) with its radius of curvature centered on another object (component 2), and another fainter image (component 5) on the opposite side, strongly supports the gravitational lens hypothesis, i.e., that component 2 is a foreground galaxy and components 1 and 5 are images of a single background object. Under this hypothesis, the multiple imaging and the arclike morphology and high inferred luminosity of component 1 result from distortion and magnification by the gravitational potential of the foreground component 2. Components 3 and 4 are other galaxies along the line of sight, possibly related to the galaxy that is component 2 and probably involved in the lensing.

The high resolution of the HST image makes the arc morphology and component 5 readily apparent and allows us to directly measure the ratio of the brightnesses of these components. This morphology and ratio are crucial elements in the development of a lens model for the source. We find additional support for the lens hypothesis from the observed morphology of component 2 . In particular, as shown in Appendix A, component 2 has the surface brightness profile and spectral energy distribution expected for a foreground elliptical galaxy, and its position angle is correctly predicted by the lens model. In the following, we adopt the interpretation of FSC $10214+4724$ as a gravitationally lensed system and describe the detailed model of this system and its consequences.

\subsection{Lens Model}

In the context of a lens model, component 1 is a "straight arc" and component 5 is a "counterimage." This gravitational lens image configuration is very common; it has been found in several clusters (see Surdej \& Soucail 1993 for a review). The model for these systems is that of a source lying on or very close to a cusp in a caustic (a line of infinite magnification; see, e.g., Blandford \& Narayan 1992) in the source plane. Although the magnification of a point source lying on the caustic is formally infinite, the maximum magnification of a real object is limited by its finite angular radius $r$. Under the gravitational lens hypothesis, the total magnification of the source should be on the same order as the flux ratio of arc to counterimage, $\sim 100$ in this case. Gravitational lens models also predict that the axis ratio of the arc should be on the same order as the total magnification. The 0.7 length of the arc thus implies an observed width on the order of 0 ".007 (50 pc), or unresolved even in HST images.

In the case of lensing dominated by mass at a single redshift, the gravitational lens mapping, which takes a twodimensional angular position $\boldsymbol{x}$ on the image plane (i.e., the position observed on the sky) to a two-dimensional angular position $y$ on the source plane (i.e., the position that would be observed if there was no lens) is a gradient mapping

$$
\boldsymbol{y}=\boldsymbol{x}-\nabla_{\boldsymbol{x}} \psi(\boldsymbol{x})
$$


where $\nabla_{\boldsymbol{x}}$ is the two-dimensional gradient operator with respect to angular image-plane position $\boldsymbol{x}$, and $\psi(x)$ is a scaled, projected, two-dimensional gravitational potential. The potential is related to the angular surface density $\Sigma$ (mass per unit solid angle)

$$
\Sigma(x)=\frac{c^{2}}{8 \pi G} \frac{D_{d} D_{s}}{D_{d s}} \nabla_{x}^{2} \psi(x),
$$

where $D_{d}, D_{s}$, and $D_{d s}$ are angular diameter distances from observer to lens (deflector), observer to source, and lens to source, and $\nabla_{x}^{2}$ is the two-dimensional Laplacian operator.

Where not otherwise stated, the lens models that follow assume that the potential $\psi$ can be approximated with a quasi-isothermal sphere with ellipticity (see, e.g., Kochanek 1991); i.e.,

$$
\psi(x)=b \sqrt{s^{2}+r^{2}}\left[1-\gamma \cos 2\left(\theta-\theta_{\gamma}\right)\right],
$$

where $x=(r, \theta)$ is the position of the point in question relative to the center of the mass distribution, $b$ is the asymptotic critical radius (the radius of the Einstein ring), roughly the angular radius of the circle of images $\left(\sim 1^{\prime \prime}\right.$ in this system because that is the angular separation of arc and lens), $\gamma$ is an ellipticity parameter, $\theta_{y}$ is the position angle of the major axis, and $s$ is a core radius. The results do not depend strongly on the core radius $s$, so it is assumed to be zero. The critical radius $b$ can be related to a one- dimensional velocity dispersion for the lens by

$$
\sigma_{v}^{2}=\frac{c^{2}}{4 \pi} \frac{D_{s}}{D_{d s}} b,
$$

although this depends on the assumption of isothermality. More secure is the mass $M$ inside the "circle of images" (in this case a circle of angular radius $b$ around component 2 ),

$$
M=\frac{c^{2}}{4 G} \frac{D_{d} D_{s}}{D_{d s}} b^{2} .
$$

The mass $M$ and the inferred luminosity $L$ of the lens can be used to compute a mass-to-light ratio as well. The inferred physical properties of the lens depend strongly on lens and source redshifts and weakly on world model. In this system the lens redshift is unknown, so $\sigma_{v}, M$, and $M / L$ are given in Figure 4 as a function of lens redshift for the model adopted below. Further discussion of Figure 4 is deferred until $\S 4.3$.

Model parameters $b, \gamma$, and $\theta_{\gamma}$ were varied to minimize the scatter in the source plane positions corresponding to the brightest pixels in the arc and counterimage, i.e.,

$$
\chi^{2} \equiv \sum_{i}\left(\Delta x_{i}\right)^{2},
$$

where the sum is over the brightest 96 pixels in the deconvolved arc and the brightest pixel in the counterimage (Fig. $1 d$ ), and $\Delta \boldsymbol{x}_{i}$ is the two-dimensional displacement on the image plane through which pixel $i$ would need to be moved

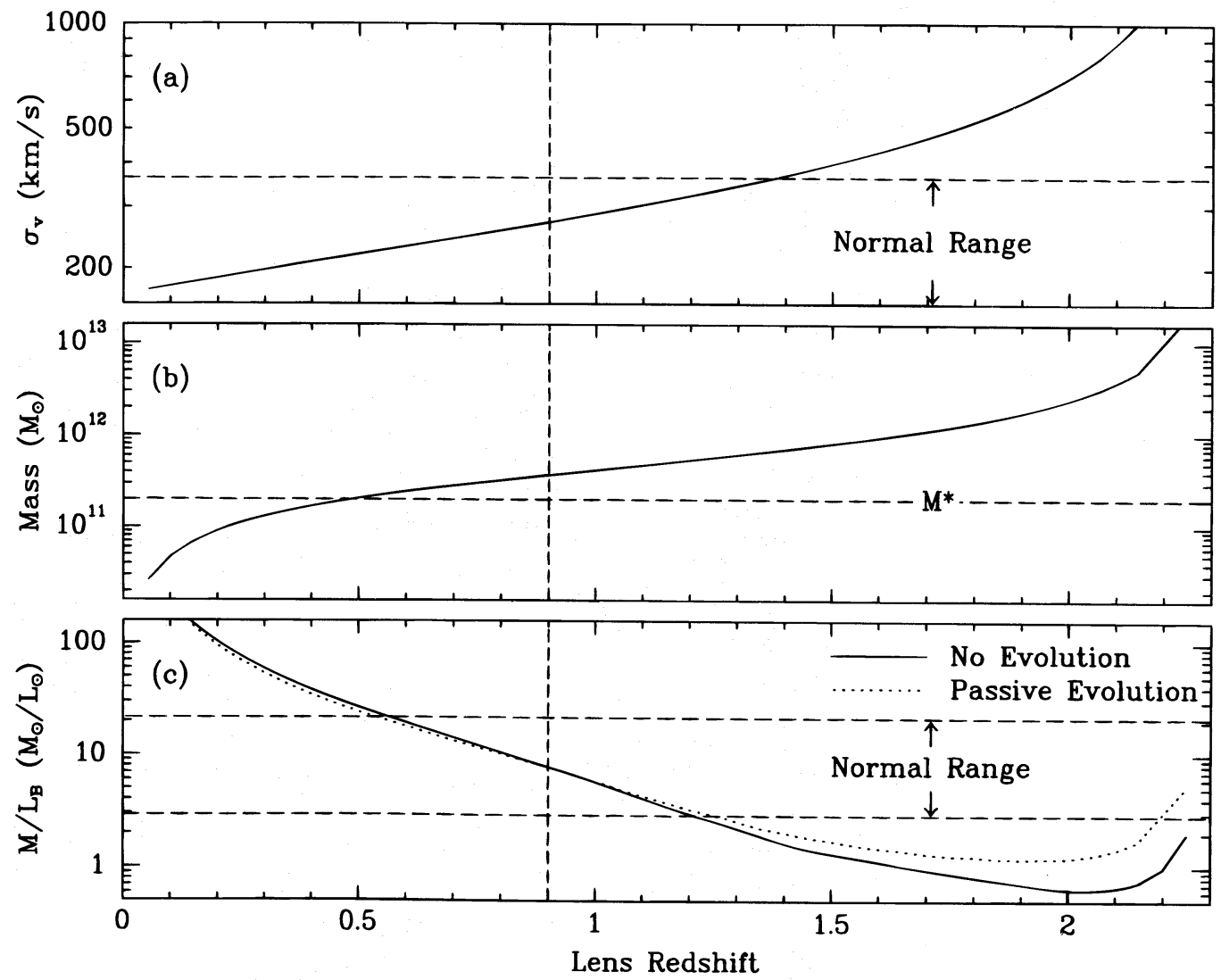

FIG. 4.-Predictions from the lens model (see $\S 4.1$ and Table 2) for the mass inside the critical radius (panel $b$ ) and the velocity dispersion (panel $a$ ) of the lens as a function of its redshift. Panel $c$ shows the mass-to-luminosity ratio using the $H S T$ F814W flux inside 0.85 radius, $K$-corrected to rest frame $B$ using unevolving (solid curve) and passively evolving (dotted curve) elliptical model spectra from Bruzual \& Charlot (1993). The dashed vertical line indicates the best estimate for the redshift of component 2 as discussed in the Appendix. Horizontal dashed lines show the normally observed range of $(M / L)_{B}$ and $\sigma_{v}$ from Fisher, Illingworth, \& Franx (1995), van der Marel (1991), and Davies et al. (1983). The value $M^{*}=2 \times 10^{11} M_{\odot}$ is from van der Marel's mean $(M / L)_{B}=6$ $M_{\odot} / L_{\odot}$ and $L_{B}^{*}=-21 \mathrm{mag}$ from Binggeli et al. (1988). The values shown are for the assumed cosmology $\left(H_{0}=50 \mathrm{~km} \mathrm{~s}^{-1}, q_{0}=0.5\right)$ and are relatively insensitive to $q_{0}$. The mass scales as $h^{-1}$ and the mass to luminosity ratio as $h$ (for the unevolving $K$-correction), while the velocity dispersion is independent of $h$, where $h$ is the Hubble constant in units of $100 \mathrm{~km} \mathrm{~s}^{-1} \mathrm{Mpc}^{-1}$. 
TABLE 2

ISOTHERMAL Lens MODEL Parameters

\begin{tabular}{lccc}
\hline \hline Parameter & Model Value & Observed & Comments \\
\hline$b \ldots \ldots \ldots \ldots \ldots \ldots \ldots$ & 0.82 & $\ldots$ & $\ldots$ \\
$\gamma \ldots \ldots \ldots \ldots \ldots \ldots \ldots$ & 0.12 & $\ldots$ & Defined by eq. (3) \\
$\epsilon \ldots \ldots \ldots \ldots \ldots \ldots \ldots$ & 0.30 & $0.16 \pm 0.1$ & $1-b / a$ \\
$\theta_{\gamma} \ldots \ldots \ldots \ldots \ldots \ldots \ldots$ & $-11^{\circ}$ & $3^{\circ} \pm 15^{\circ}$ & E of $\mathrm{N}$ \\
$b_{3} \ldots \ldots \ldots \ldots \ldots \ldots$ & $0^{\prime \prime} 60$ & $\ldots$ & $\ldots$ \\
Source radius $\ldots \ldots$ & $0^{\prime \prime} 0055$ & $\ldots$ & For magnification ratio 100 \\
\hline
\end{tabular}

Note.-The model assumes the lensing potential arises from components 2 and 3, and that these components have isothermal potentials and the same redshift and mass to $(K$-band) light ratios. The ellipticity $\epsilon$ is the conventional value defined by 1 minus the ratio of semiminor to semimajor axis and differs from the model ellipticity parameter $\gamma$, which is defined by eq. (3). The "source radius" is the angular radius at which, for a circular source, the arc-counterimage magnification ratio is 100 . Other symbols are explained in the text. World model $q_{0}=0.5$ is assumed. Changing world models only changes the numbers by $\sim 10 \%$.

in order for it to project (via the lens mapping) to the same location on the source plane as that of the brightest pixel in the arc. Image-plane rather than source-plane displacements were used for computing the scatter because the image plane is the observed plane, the plane on which uncertainties are homogeneous and isotropic. On the source plane the uncertainties have been mapped through the nonlinear lens mapping and are extremely inhomogeneous and anisotropic. The minimum rms scatter of the pixels (in image plane coordinates) was $0.7 \mathrm{PC}$ pixels.

The best-fit model parameters are given in Table 2. The inferred intrinsic source radius that makes the arccounterimage flux ratio 100 is 0 ".0055 (44 pc). The model makes the assumption that component 3 is a singular isothermal sphere $(\gamma=s=0)$ at the same redshift as component 2 and with critical radius $b_{3}=0{ }^{\prime \prime} 6$, the expected value under the assumption that components 2 and 3 have the same mass to $(K$-band) light ratio. A simpler model, which assumes that the potential is entirely due to an elliptical shaped mass centered on component 2 , was also considered. The two-component model was adopted because the intrinsic ellipticity of the potential in this model is smaller than in the simpler model, in better agreement with the observed ellipticity in component 2 . This is because the external mass of component 3 has a tidal effect that replaces some of the ellipticity in the primary lens. ${ }^{4}$ The predicted orientation of the lens in the models is consistent with the observed orientation of component 2 . Figure $1 f$ shows the density and potential contours for the adopted model, as well as the critical curve and the image morphology for a circular source of radius 0.0055 , smoothed to a FWHM of 0 . 02 , and with the counterimage brightness enhanced for visibility. Figure $1 e$ shows the model image morphology convolved with the synthetic PSF discussed in $\S 3$ and should be compared to Figure 1c.

The image configuration in the lens model is that of a triple image or straight arc (plus counterimage). Although parts of the source are triply imaged in component 1 , the source radius inferred from the flux ratio of components 1 and 5 is large enough that the three images merge into a single straight arc. We interpret the peak in the east half of the arc as corresponding to two images merging on the

\footnotetext{
${ }^{4}$ If component 3 is at a larger redshift than component 2 (see the Appendix) the agreement in ellipticity is slightly better yet, but we adopt a single redshift for components 2 and 3 to confine the number of parameters.
}

critical curve, while the peak in the west half corresponds to the third image. The triple structure may become more apparent in high-resolution images in other bandpasses if the flux at those wavelengths is produced by structures offset by $\sim 0 " 02(160 \mathrm{pc})$ from those that produce the F814W flux or having intrinsic size scales a factor of $\sim 3$ smaller.

The source location near the point at which three images on one side of the lens merge into a single image causes high magnification. As discussed by Broadhurst \& Lehár (1995), the magnification is thus a sensitive function of source size and position. The inferred size and position in turn depend on the assumption of an isothermal profile for the lens potential, i.e., $\psi \propto r$. For a shallower potential, $\psi \propto r^{0.9}$, the best-fit model puts the center of the source further inside the three-image region than for the isothermal case, and the inferred source radius from the arc-counterimage flux ratio is $0^{\prime \prime} 013$. For $\psi \propto r^{1.1}$, the inferred source radius is $0^{\prime \prime} 0046$.

The predicted total magnification of $\mathrm{F} 814 \mathrm{~W}$ emission from a uniform circular source as a function of source radius is shown for all three potential models in Figure 5. In each case, the total magnification for the source radius derived above from the flux ratio of component 1 to component 5 (i.e., 100) is less or greater than 100 because component 5 is somewhat demagnified or magnified. The dependence of the calculation of the total magnification in the HST image on the assumption of a circular source geometry for the F814W emission was investigated for the isothermal model. Sources of the same total projected solid angle on the sky have the same total magnifications to within $\sim 15 \%$ even if they are highly elliptical, no matter what their position angle. The magnification in the isothermal model scales as $r^{-1}$ for very small sizes or separations from the caustic and smoothly converts to $r^{-0.5}$ at larger radii, in agreement with Schneider, Ehlers, \& Falco (1992), and can be approximated to $\sim 20 \%$ by $\mathrm{M}=3.9 r^{-0.624}$ for the range $0.001<r<1^{\prime \prime}(8-8000 \mathrm{pc})$. The kink at $\sim 0^{\prime \prime} .005$ in Figure 5 for $\psi \propto r^{0.9}$ occurs where the source size becomes large enough to make the three distinct images merge into a single arc. Because of the fact that in the other two models the source location is closer to the point at which the three images merge, the source radii at which the mergers take place are too small to appear in Figure 5. The bump near $r \sim 0.5$ in Figure 5 corresponds to the formation of a ring (see below).

Different distributions for the narrow line and UV and optical continuum regions, and the likelihood of substantial 


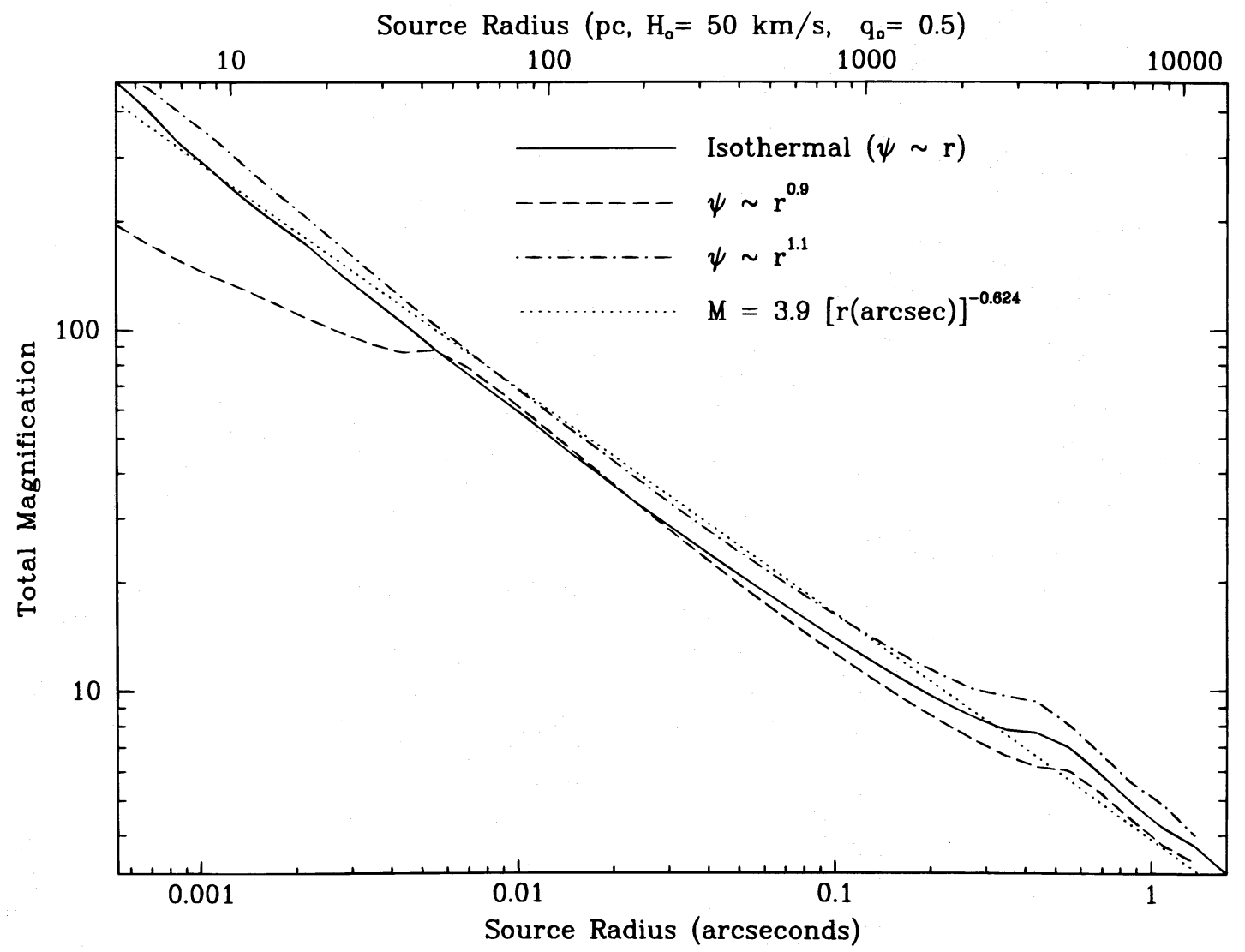

Fig. 5.-Predictions from the lens model for the total magnification $\mathbf{M}$ of the background source flux, assuming a uniformly illuminated source of radius $r$ (arcseconds, shown on the bottom axis) or $R$ (parsecs, shown on the top axis). Different line types correspond to different assumed potentials as shown in the legend. The dotted line shows a power-law approximation to the predicted magnification.

reddening (Elston et al. 1994), can therefore account for the substantially different appearance of FSC $10214+4724$ at different wavelengths noted by Matthews et al. (1994), and in particular for the larger extent of the $K$-band arc seen by Matthews et al. and Graham \& Liu (1995) than the arc seen in the $H S T$ image. The $140^{\circ}$ extent of the $K$-band arc corresponds to a source with $0.25(2 \mathrm{kpc})$ radius. If the source radius is increased to $\sim 0$ ". 5 , it is imaged into an elliptical $(\epsilon \sim 0.4)$ ring connecting components 1 and 5 . The position angle of this ring is perpendicular to that of component 2 and is offset from being perfectly centered on component 2 by $\sim 0$.". in the direction of component 1 . The excess light near 1".4 radius noted in $\S 3.2$ may be the UV (rest frame) counterpart of the more extended arc seen in the $K$ images. Note that Matthews et al. find the $\mathrm{H} \alpha$ emission to be extended in an east-west direction by $\sim 0$."5, which suggests that the narrow-line region is largely coincident with the UV continuum that dominates the $\mathrm{F} 814 \mathrm{~W}$ image.

\subsection{Bolometric Luminosity of FSC $10214+4724$}

FSC $10214+4724$ has an apparent luminosity of $L_{\text {app }}=$ $5 \times 10^{14} L_{\odot}$ (Rowan-Robinson et al. 1993), making it among the most luminous known objects in the universe. The vast majority of this luminosity, $\sim 99 \%$, is observed in the infrared (Rowan-Robinson et al. 1991, 1993). There is strong evidence that the UV source is a quasar (FWHM of C III] $\sim 10,000 \mathrm{~km} \mathrm{~s}^{-1}$ in polarized light; Goodrich et al. 1996) enshrouded in dust $\left(\mathrm{H} \alpha / \mathrm{H} \beta \geq 20\right.$, implying $A_{V}>5.5$; Elston et al. 1994), and that the quasar's luminosity is absorbed in the dust shell and reradiated in the infrared (Rowan-Robinson 1993). This implies that the size of the infrared emitting region is substantially larger than the optical/UV emitting region.

If FSC $10214+4724$ is magnified by a gravitational lens, the intrinsic source luminosity is less than the apparent luminosity. However, if the infrared source is larger than the optical/UV source, the magnification of the infrared source is less than the magnification measured from the $H S T$ image. The magnification of the infrared source can be estimated by assuming that the infrared source can be approximated as an optically thick blackbody. This assumption corresponds to making the infrared source as small as possible and hence the magnification of the infrared radiation as large as possible. In this case, because of the assumption that the emitted infrared energy distribution is independent of distance from the central heating source, the magnification is independent of wavelength. The temperature of the dust is assumed to be $T \sim 140 \mathrm{~K}$. At this temperature the emission peaks at a rest wavelength of $18 \mu \mathrm{m}$, which corresponds to the observed emission that peaks at $60 \mu \mathrm{m}$.

With this temperature, the apparent luminosity $L_{\text {app }}$ and intrinsic luminosity $L_{\text {int }}$ can be written as

$$
L_{\mathrm{app}}=\mathrm{M}(R) L_{\mathrm{int}}=\mathrm{M}(R) \times 4 \pi R^{2} \sigma T^{4},
$$

where $R$ is the physical radius of the source, $\mathrm{M}(R)$ is the magnification from Figure 5 for a uniform disk of radius $R$, and $T$ is the blackbody temperature determined by the wavelength of peak emission. Solving this equation for $R$ gives a radius of $130 \mathrm{pc}\left(0^{\prime \prime} .017\right)$, and $\mathrm{M}(R)=42$ for the isothermal model, so that the intrinsic luminosity of FSC $10214+4724$ is $1.2 \times 10^{13} L_{\odot}$. A somewhat larger source size and lower magnification is derived if the temperature $T$ 
is assumed to be $115 \mathrm{~K}$, the color temperature determined by the observed flux densities at 60 and $450 \mu \mathrm{m}$ and corrected for redshift. Then the radius of the infrared source is $240 \mathrm{pc}(0.03)$, the magnification is 29 , and the intrinsic luminosity is $1.7 \times 10^{13} L_{\odot}$. Note that at these source radii the magnification is not very sensitive to the assumed potential (see Fig. 5).

The expected arc length is $\sim 2 r \mathrm{M}(r)$, or $1^{\prime \prime} .7$ in the $T=115 \mathrm{~K}$ case, and 1".4 for $T=140 \mathrm{~K}$. From VLA-A configuration observations at $8.4 \mathrm{GHz}$ with 0 ".25 resolution, Lawrence et al. (1993) found a 0".6 (east-west) by 0"3 source. The similarity of this structure to the arc in the HST image suggests a continuum radio source radius closer to the 0 ".005 (40 pc) estimated for the optical/UV source than to the minimum infrared source size just calculated. Condon et al. (1991) find that the radio source size for nearby IRAS galaxies with infrared luminosities greater than $10^{12} L_{\odot}$ is typically $\sim 100$ pc (and for Mrk 231, the most luminous of the sample, $\lesssim 1 \mathrm{pc}$ ), smaller than the minimum blackbody size for far-infrared emission from these galaxies. For their sample Condon et al. find $\langle q\rangle=2.34$, where $q$ is the logarithm of the ratio of far-infrared $(60-100 \mu \mathrm{m})$ to 1.49 $\mathrm{GHz}$ flux. For FSC 10214+4724, extrapolating the Lawrence et al. (1993) observed radio flux to $0.45 \mathrm{GHz}$ (the observed frequency for emitted $1.49 \mathrm{GHz}$ ) yields $3.5 \mathrm{mJy}$, and interpolating to the rest frame wavelengths for 60 and $100 \mu \mathrm{m}$ and using the definition of Condon et al. gives $q=1.91$. If the radio magnification is 100 and the farinfrared magnification is 30 , then the intrinsic $q=2.39$. Therefore the radio morphology and flux measured by Lawrence et al. are quite consistent with the above estimate for the bolometric luminosity.

The 0 ". 6 extent of the radio morphology is also consistent with a much smaller radio continuum source size, although the value of $q$ would then be significantly larger than observed for local luminous IRAS galaxies. It would be interesting (albeit quite challenging) to see whether the very high angular resolution possible with VLBI observations revealed the triple structure in the arc discussed above.

The size of the infrared source determined under the assumption of optically thick emission is a plausible lower limit to the physical source size. Alternatively, the magnification can be estimated based on the models of Phinney (1989) of infrared emission from dusty, warped disks illuminated by a central quasar. The physical size of the source required to obtain a self-consistent solution for the intrinsic luminosity is quite large. For the region emitting at $150 \mu \mathrm{m}$ (450 $\mu$ m observed), the source radius would be $\sim 10^{\prime \prime}$, much larger than the observed size of the CO source (see, e.g., Scoville et al. 1995). Thus we consider such a model less consistent with the observations than the optically thick models.

The reduction in the intrinsic luminosity of FSC $10214+4724$ to $\sim 2 \times 10^{13} L_{\odot}$ implied by the lens model of the source brings it into the luminosity range of previously studied infrared luminous active galactic nuclei. The $I R A S$ source FSC $15307+3252$ at a redshift of $z=0.93$ has a luminosity of $4 \times 10^{13} L_{\odot}$, while the IRAS source PSC $09104+4109$ has a luminosity of $2 \times 10^{13} L_{\odot}$ for our assumed cosmology (Cutri et al. 1994). There is no known evidence from high-resolution imaging (Soifer et al. 1994, 1995; Hutching \& Neff 1988) that either of these sources is a gravitational lens, so the apparent luminosity is presumably the intrinsic luminosity in these cases. Thus, based on its bolometric luminosity, FSC $10214+4724$ is most likely a source similar to these. The reduction in intrinsic luminosity reduces the necessary dust mass associated with the source (Rowan-Robinson et al. 1993) by the same magnification factor, into the range $M_{\text {dust }} \sim 1-3 \times 10^{7} M_{\odot}$, which is consistent with the estimates of the gas mass based on the dynamical mass determinations from the $\mathrm{CO}$ observations (Scoville et al. 1995).

\subsection{Properties of Component 2}

No conclusive measurement of the redshift for component 2 has yet been made, to our knowledge, although tentative values of 0.42 (Close et al. 1995) and 0.90 (Serjeant et al. 1995) have been suggested based on possible continuum breaks in the spectrum of component 2, while Goodrich et al. (1996) find $\mathrm{Mg}$ lines in absorption at $z=1.32$ (and possibly $z=0.89$ ) in the spectrum of component 1 . In the Appendix we provide three estimates of the redshift for component 2 (two of which are closely related). All three estimates are consistent with $z \sim 0.9$, and we adopt this value as our best overall estimate of the redshift. Note the SED and $R_{e}-\left\langle\mu_{B}\right\rangle_{e}$ estimates do not assume component 2 is a lens, only that it is an elliptical galaxy, and therefore give additional support to the lensing hypothesis by placing component 2 at an intervening redshift relative to FSC $10214+4724$.

The velocity dispersion $\sigma_{v}$, mass $M$, and mass-to-light ratio $(M / L)$ predicted for the lens are shown in Figure 4 as a function of lens redshift. Adopting $z=0.9$ yields $(M / L)_{B}=8$ $M_{\odot} / L_{\odot}$ (vs. the observed average of $6 M_{\odot} / L_{\odot}$; van der Marel 1991), $\sigma_{v}=270 \mathrm{~km} \mathrm{~s}^{-1}$, and $M=3.9 \times 10^{11} M_{\odot}$ (thus $L_{B}=5 \times 10^{10} L_{\odot}$ ). These values are for a radius of 0 . 85 : using Figure 3 the total blue luminosity is then $L_{B}=$ $1.4 \times 10^{11} L_{\odot}$ or $\sim 4 L^{*}$ (Binggeli, Sandage, \& Tammann 1988). These values are independent of evolutionary model because $\mathrm{F} 814 \mathrm{~W}$ samples rest frame $B$ at $z=0.9$. The velocity dispersion and mass estimated by Graham \& Liu (1995), Broadhurst \& Lehár (1995), and Close et al. (1995) are consistent with Figure 4, but their total luminosity is lower [and hence $(M / L)_{B}$ higher] because a smaller aperture correction than is shown in Figure 3 was assumed.

Thus for the redshift estimate $z=0.9$ the present lensing model predicts properties typical of present-day elliptical galaxies, except that the galaxy is unusually luminous. The probability of a large lensing galaxy is greater than the galaxy luminosity function alone implies, however, because the cross section for gravitational lensing is proportional to mass.

\subsection{The Parent Population of IRAS FSC $10214+4724$}

Analysis of statistically complete samples of radio galaxies suggests that the lensing rate (i.e., probability that a given radio galaxy is lensed) is on the order of $1 / 500$ (Miralda-Escudé \& Lehár 1992; Myers et al. 1995). Given that a source is lensed, the probability of getting total magnification $\mathrm{M}$ is on the order of $M^{-2}$ (e.g., Schneider et al. 1992). The estimated total magnification $\sim 30$ for the $I R A S$ flux from $\S 4.2$ corresponds to a likelihood of $\sim 10^{-3}$. The existence of a single lensed object in the surveyed area $(0.2$ sr; Rowan-Robinson 1991) with magnification 30 should, according to these probabilities, represent an underlying population of $\sim 800$ compact, $60 \mu \mathrm{m}$ luminous objects per square degree (or $>40$ per square degree at $95 \%$ confidence) that are either not lensed or lensed with much 
lower magnification (and hence are not in the FSS catalog). If they are like IRAS FSC $10214+4724$, these sources will have observed magnitude $r \sim 25 \mathrm{mag}$, and their IR fluxes will be of order $3 \mathrm{mJy}$ at $25 \mu \mathrm{m}$ and $7 \mathrm{mJy}$ at $60 \mu \mathrm{m}$. To these flux levels, models of the IR galaxy population with strong luminosity evolution (Hacking \& Soifer 1991) predict a few hundred sources per square degree, in agreement with this estimate. Of course this is only an order of magnitude estimate because it depends on extrapolation from a single serendipitously discovered object, and on the relative redshift distributions of IR-luminous and radio galaxies. Optical field galaxy redshift surveys now underway with the Keck Telescope are approaching this depth (J. Cohen, private communication; UC DEEP collaboration, private communication), and IR imaging surveys to well beyond these levels are envisioned with ISO, WIRE, and SIRTF, so this very uncertain prediction may be testable in the near future.

\section{SUMMARY}

We have obtained a $0.8 \mu \mathrm{m}$ image of the $z=2.286 I R A S$ source FSC $10214+4724$ with the HST WFPC2 Planetary Camera, with 0 ". 1 resolution and high signal to noise. We find the following:

1. The source appears as an unresolved ( $<0$ ".06 wide) arc 0.7 long, with significant substructure along its length. The arc is roughly centered on a galaxy 1 ".18 to the north (component 2), and a faint unresolved component (component 5) is clearly detected 0 ".43 north of component 2. Two other galaxies (components 3 and 4) are evident within a few arcseconds of the IRAS source. This morphological configuration is characteristic of a gravitationally lensed system, in which the arc and component 5 are images of a single background source produced by the potential of the foreground component 2.

2 . The surface brightness profile of component 2 is well matched by a de Vaucouleurs profile, characteristic of an elliptical galaxy with an effective radius of 1 .27. There is evidence for excess emission above the de Vaucouleurs profile near the radius of the arc.

3. The flux ratio of the arc to component 5 is $\sim 100$, implying magnification in the HST image of the background source by roughly this amount.

4. A detailed lensing model, which reproduces the observed morphology and relative flux of the arc and counterimage, correctly predicts the position angle for component 2 . Better agreement is found with the observed ellipticity of component 2 if component 3 is included in the lensing potential. The model predicts reasonable values for the mass and velocity dispersion of component 2 .

5. If component 2 is an elliptical galaxy, its spectral energy distribution is inconsistent with it being at $z=2.286$, and $z=0.9$ is preferred. The surface brightness profile of component 2 implies a redshift between 0.6 and 1.2. From the lensing model, for $z \sim 0.9$, the central mass-to-light ratio for component 2 is $(M / L)_{B}=8 M_{\odot} / L_{\odot}$, the velocity dispersion $\sigma_{v}=270 \mathrm{~km} \mathrm{~s}^{-1}$, and the total blue luminosity $L_{B}=1.4 \times 10^{11} L_{\odot} \sim 4 L^{*}$.

6. The model predicts an intrinsic radius of $\sim 0$.005 (40 pc) for the background source at $0.25 \mu \mathrm{m}$ rest wavelength. Triple structure in the arc is obscured by this source size but may become apparent at high resolution in other bandpasses. The larger size of the arc observed at $K$ implies an intrinsic source radius of 0.25 in the corresponding emitting bandpass. A source of radius greater than 0.5 would produce a ring of emission connecting the arc and component 5 . This may account for the excess emission seen in the surface brightness profile of component 2. The $\mathrm{H} \alpha$ and radio continuum morphologies appear similar to that of the $0.8 \mu \mathrm{m}$ arc, implying a similar source size for the narrowline, UV continuum, and radio continuum emission.

7. The minimum source size for an optically thick blackbody source producing the bulk of the bolometric luminosity is $\sim 0$.03 $(240 \mathrm{pc})$, implying a bolometric magnification of $\sim 30$. The background lensed source then has an intrinsic luminosity $\sim 2 \times 10^{13} L_{\odot}$. Thus IRAS FSC $10214+4724$ is not the most luminous object in the universe, but it remains among the most luminous in the IRAS catalog.

8. The expected incidence of 30 fold gravitational magnification is low enough to suggest that FSC $10214+4724$ represents an underlying population of $\sim 800$ compact objects per square degree with optical magnitude $r \sim 25$ mag and $F_{60 \mu \mathrm{m}} \sim 7 \mathrm{mJy}$.

We thank Mark Dickinson for help with the $R_{e}-\left\langle\mu_{B}\right\rangle_{e}$ technique for estimating $z$ and in particular for supplying the Sandage \& Perelmutter data in electronic form, Adam Stanford for calculating K-corrections and general assistance with STSDAS, and Roger Blandford for help with lens modeling. We acknowledge helpful discussions with James Graham, Michael Liu, Tom Broadhurst, Joseph Lehár, and Joseph Miller. The ideas of rotating (rather than merely flipping) component 2 in $\S 3.2$ and of a magnification versus radius plot (cf. Fig. 5) were suggested by Broadhurst and Lehár. An anonymous referee reminded us of the sensitivity of the derived magnification to the isothermal profile assumption. This research was supported by NASA through a grant awarded by STScI, which is operated by AURA under NASA contract NAS 5-26555. Portions of the research described in this paper were carried out by the Jet Propulsion Laboratory, California Institute of Technology, under a contract with NASA.

\section{APPENDIX A}

\section{ESTIMATES OF THE REDSHIFT FOR COMPONENT 2}

Here we use the spectral energy distribution and surface brightness profile of component 2 to estimate its redshift.

\section{A1. SPECTRAL ENERGY DISTRIBUTION}

Figure 6 combines $r$ and $H$ data from Elston et al. (1994), $J$ and $K$ from Matthews et al. (1994), and F814W data from the present work for components 2,3 , and 4 , normalized at $K$. (Note that component 1 in the Elston et al. terminology is our 

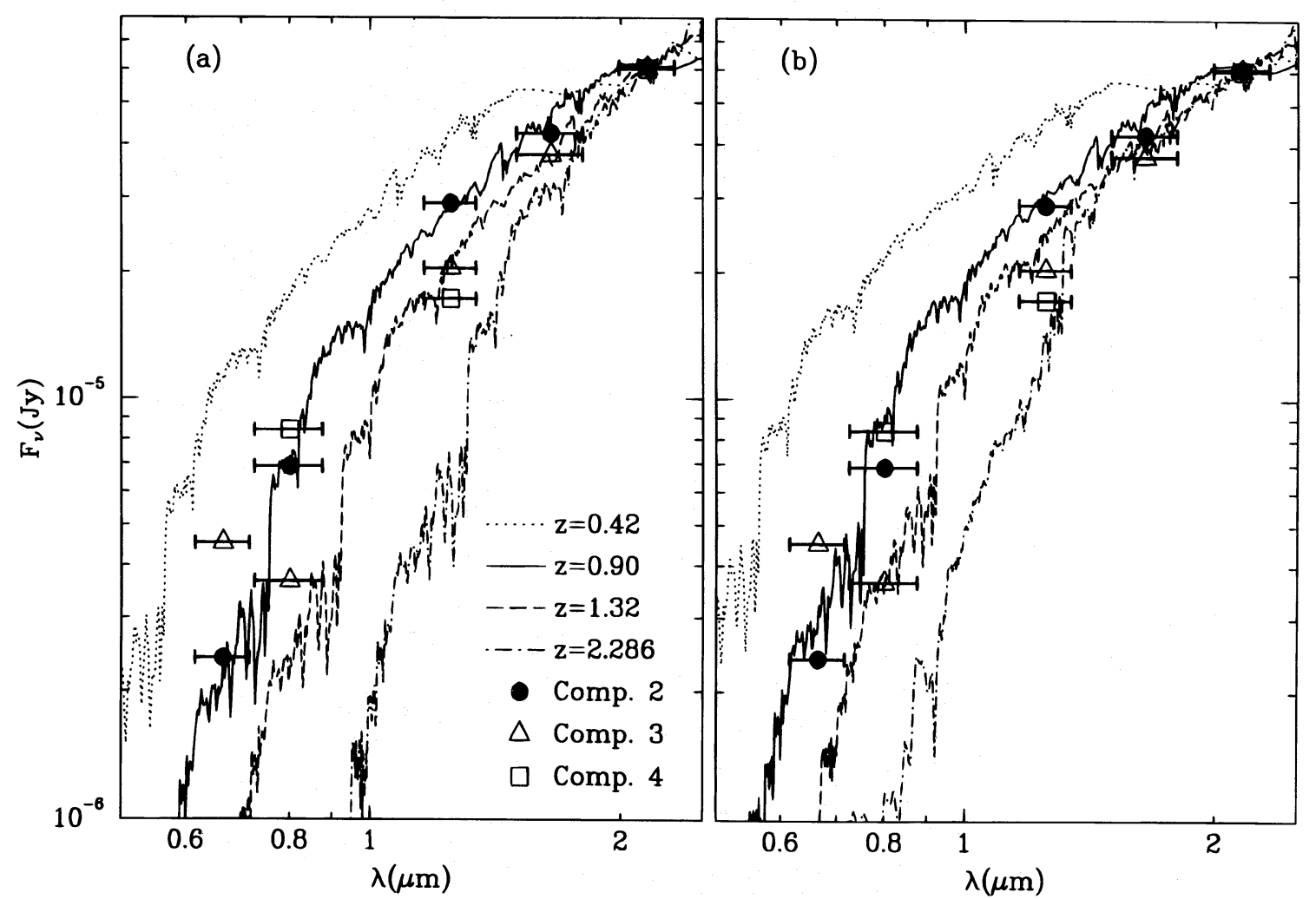

FIG. 6.- Spectral energy distributions for components identified in Fig. 1b, with unevolving $(a)$ and passively evolving $(b)$ model elliptical spectra from Bruzual \& Charlot (1993) shown for comparison. The component 2 data fit the $z=0.9$ models well. The flux scale is correct for component 2 ; for component 3 it should be reduced by a factor of 2.3 and for component 4 by a factor of 9 . The data are derived from Elston et al. (1994), Matthews et al. (1994), and this paper. See $\S$ A1 for further details.

component 2.) Because the angular resolution of the three datasets ranges from $0.1-1.5$, the combined spectral energy distribution (SED) is somewhat uncertain.

Given the good agreement of the surface brightness profile for component 2 with a de Vaucouleurs law (Fig. 3), it is reasonable to assume that this component is an elliptical galaxy. For comparison, the unevolved spectrum of a standard Bruzual \& Charlot (1993) elliptical galaxy model at an age of $13 \mathrm{Gyr}$ and redshifts of $0.42,0.90,1.32$, and 2.286 is plotted in Figure $6 a$; the corresponding passively evolving model with ages of 7.75, 5, 3.75, and $2.2 \mathrm{Gyr}$ at these redshifts (the ages are consistent with a present age of $13 \mathrm{Gyr}$ with the assumed cosmology) is plotted in Figure $6 \mathrm{~b}$. All models were normalized to the $K$ flux in the SED for component 2. Clearly the $z=2.286$ models fail to match the observed SED for component 2, while the $z=0.9$ models provide surprisingly good fits to the observations. This is fairly strong evidence that component 2 is in fact a foreground elliptical: it is too blue to be an elliptical galaxy at the redshift of FSC 10214+4724.

The $r$ data point for component 3 is anomalously bright, while the rest of its SED is somewhat redder than component 2. This might be due to a combination of reddening and star formation associated with the tidal interaction suggested in $\S 3.1$, placing component 3 at the same redshift as component 2 , as the adopted model in $\S 4.1$ assumes. Alternatively, the $z=1.32$ SED models appear to fit component 3 at least as well as the $z=0.9$ models. Without the constraint of an elliptical surface brightness profile, it is much more difficult to assign a unique redshift based on the SED for component 3 than for component 2. Putting component 3 at $z=1.32$ implies a higher lensing mass, for a constant mass-to-light ratio, and the result is a decrease in the mass and ellipticity of component 2 by of order $10 \%$. Component 4 is significantly bluer than components 2 and 3 , consistent with what appears to be a later type morphology.

\section{A2. FUNDAMENTAL PLANE RELATIONS}

Since the surface brightness profile of component 2 strongly suggests it is an elliptical galaxy ( $\$ 3.2$ and Fig. 3 ), it is possible to make further use of the surface brightness profile to estimate the redshift of component 2 using the fundamental plane relations for ellipticals (Kormendy \& Djorgovski 1989). Using $r_{e}=1$ '.274 and $m_{814}=21.04$ within $r_{e}$ from Figure 3, the present-day equivalent blue surface brightness of component 2 was calculated by correcting for $(1+z)^{4}$ surface brightness dimming, redshift $K$-correction, and luminosity evolution. Figure 7 shows $R_{e}$ (in kiloparsecs) and $\left\langle\mu_{B}\right\rangle_{e}$ (the average blue surface brightness within $R_{e}$ ) as a function of the assumed redshift for component 2, overlaid on the data for present-day ellipticals from Sandage $\&$ Perelmutter (1990). Luminosity and $K$-corrections are shown for both a nonevolving and passively evolving elliptical model spectrum from Bruzual \& Charlot (1993). Redshifts near zero, or in the range 0.6-1.2, can be accommodated. From Figure 4, the lens model predicts a central mass-to-light ratio greater than 100 for $z<0.2$, arguing against low values. We consider the passively evolving model more realistic, leading to an estimate of $z=1.0 \pm 0.2$. This estimate is independent of $H_{0}$ because the present-day data scale in the same way as the calculated values. The estimate is 


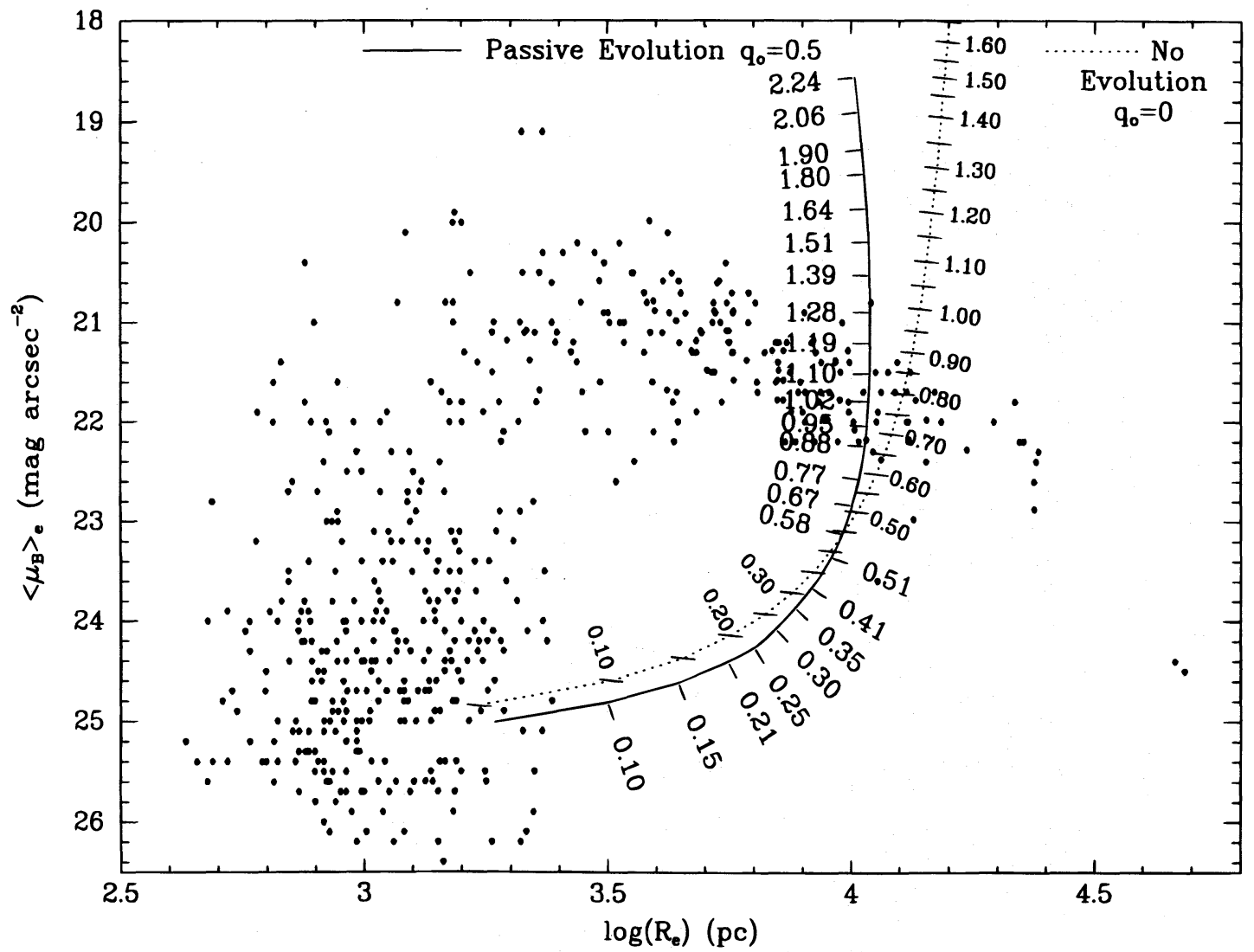

Fig. 7.-Estimate of the redshift for component 2 using the average blue surface brightness-effective radius relation for giant elliptical galaxies. The data are from Sandage \& Perelmutter (1990). The dotted curve shows calculated $\left\langle\mu_{B}\right\rangle_{e}$ and $R_{e}$ values with $K$-corrections from an unevolving Bruzual \& Charlot (1993) elliptical model with $q_{0}=0$ and a uniform redshift interval of 0.05 . Here $\left\langle\mu_{B}\right\rangle_{e}$ is the average blue surface brightness within the effective radius. The solid curve is for a passively evolving elliptical and $q_{0}=0.5$.

driven almost entirely by the $(1+z)^{4}$ dependence of surface brightness on redshift and is relatively independent of $q_{0}$ because the latter primarily affects angular size, which is nearly orthogonal to redshift in the region of interest in Figure 7 . The main uncertainty is due to luminosity evolution (some of which arises from the dependence of timescales on $q_{0}$ ) and to the scatter in surface brightness among giant elliptical galaxies.

Much of this scatter is correlated with the velocity dispersion, and if $\sigma$ is known the $D_{n}-\sigma$ relation (Lynden-Bell et al. 1988) can be used to measure the angular diameter distance for giant elliptical galaxies. Here $D_{n}$ is defined as the angular diameter of the circle within which the integrated rest-frame blue surface brightness is $20.75 \mathrm{mag} \mathrm{arcsec}-2$ after correction for luminosity evolution and $(1+z)^{4}$ surface brightness dimming. The value of $\sigma$ is found from the lens model as plotted in Figure $4 a$. An advantage of $D_{n}$ over $R_{e}$ is that $D_{n}$ is defined at a higher surface brightness level and is therefore smaller than $R_{e}$ and more immune to uncertainties about emission at the arc radius, as well as being less sensitive to uncertainties in sky subtraction. However, the technique is sensitive to $q_{0}$ because it is essentially an angular diameter distance. The redshift estimates from this approach range from 0.75 for the case $q_{0}=0$ and no evolution, to 1.15 for $q_{0}=0.5$ and passive evolution, independent of $H_{0}$.

\section{REFERENCES}

Binggeli, B., Sandage, A., \& Tammann, G. A. 1988, ARA\&A, 26, 509 Blandford, R. D., \& Narayan, R. 1992, ARA\&A 30, 31

Broadhurst, T. J., \& Lehár, J. 1995, ApJ, 450, L41

Brown, R. L., \& Vanden Bout, P.A. 1991, AJ, 102, 1956

Bruzual, G., \& Charlot, S. 1993, ApJ, 405, 538

Clements, D. L., Rowan-Robinson, M., Lawrence, A., Broadhurst, T., \& McMahon, R. 1992, MNRAS, 256, 35P

Close, L. M., Hall, P. B., Liu, C. T., \& Hege, E. K. 1995, ApJ, 452, L9

Condon, J. J., Huang, Z.-P., Yin, Q. F., \& Thuan, T. X. 1991. ApJ, 378, 65

Cutri, R. M., Huchra, J. P., Low, F. J., Brown, R. L., \& Vanden Bout, P. A 1994, ApJ, 424, L65

Davies, R. L., Efstathiou, G., Fall, S. M., Illingworth, G., \& Schechter, P. L. 1983, ApJ, 266, 41

Downes, D., Radford, S. J. E., Greve, A., Thum, C., Solomon, P. M., \& Wink, J. E. 1992, ApJ, 398, L25

Elston, R., McCarthy, P. J., Eisenhardt, P., Dickinson, M., Spinrad, H., Jannuzi, B. T, \& Maloney, P. 1994, AJ, 107, 910

Fisher, D., Illingworth, G., \& Franx, M. 1995, ApJ, 438, 539

Goodrich, R. W., Miller, J. S., Martel, A., Cohen, M. H., Tran, H. D., Ogle,

P. M., \& Vermeulen, R. C. 1996, ApJ, 456, L9
Graham, J. R., \& Liu, M. C. 1995, ApJ, 449, L29

Hacking P. B. \& Soifer, B. T. 1991, ApJ, 367, L49

Hutchings, J. B., \& Neff, S. G. 1988, AJ, 96, 1575

Kochanek, C. S. 1991, ApJ, 373, 354

Kormendy, J., \& Djorgovski, S. 1989, ARA\&A, 27, 235

Lawrence, A, et al. 1993, MNRAS, 260, 28

Lynden-Bell, D., Faber, S. M., Burstein, D., Davies, R. L., Dressler, A., Terlevich, R. J., \& Wegner, G. 1988, ApJ, 326, 19

Matthews, K., et al. 1994, ApJ, 420, L13

Miralda-Escudé, J., \& Lehár, J. 1992, MNRAS, 259, 31P

Myers, S. T., et al. 1995, ApJ, 447, L5

Phinney, E.S. 1989, Theory of Accretion Disks, ed. W. Duschl, F. Meyer, \& J. Frank (Dordrecht: Kluwer), 457

Rowan-Robinson, M., et al. 1991, Nature, 351, 719 1993, MNRAS, 261, 513

Sandage, A., \& Perelmutter, J.-M. 1990, ApJ, 361, 1

Schneider, P., Ehlers, J., \& Falco, E. E. 1992, Gravitational Lenses (New York: Springer)

Serjeant, S., Lacy, M., Rawlings, S., King, L., \& Clements, D. L. 1995, MNRAS, 276, L31 
Scoville, N. Z., Yun, M. S., Brown, R. L., \& Vanden Bout, P.A. 1995, ApJ, 449,109

Soifer, B. T., Neugebauer, G., Armus, L., \& Shupe, D. L. 1995, AJ, in press

Soifer, B. T., Neugebauer, G., Matthews, K., \& Armus, L. 1994, ApJ, 433, L69

Solomon, P. M., Downes, D., \& Radford, S. J. E. 1992, ApJ, 398, L29

Note added in proof.-The outermost density contour in Fig. $1 f$ is at $0.5 \Sigma_{c}$, increasing to $2.5 \Sigma_{c}$ in steps of $0.5 \Sigma_{c}$, where $\Sigma_{c}$ is the mass per unit solid angle inside the critical radius $b$, or $M /\left(\pi b^{2}\right)$ in equation (5). Potential contours are in spacings of 0.62 square arcseconds.
Surdej, J., \& Soucail, G. 1993, in Proc. 31st Liège Internat. Astrophys. Colloq., Gravitational Lenses in the Universe, ed. J. Surdej et al. (Liège: Univ. of Liège), 205

Trentham, N. 1995, MNRAS, 277, 616

Tsuboi, M., \& Nakai, N. 1992, PASJ, 44, L241

van der Marel, R. P. 1995, MNRAS, 253, 710 